\title{
TITLE: Exploring potential mechanisms involved in the relationship between eudaimonic wellbeing and nature connection
}

\section{Authors}

Anne CLEARY, School of Medicine and Menzies Health Institute Queensland, Griffith University, Gold Coast, Australia, anne.cleary@ griffithuni.edu.au +61 422784441 [Corresponding author]

Kelly S. FIELDING, School of Communication and Arts, The University of Queensland, Brisbane, Australia, k.fielding@uq.edu.au

Sarah L. BELL, European Centre for Environment and Human Health, University of Exeter Medical School, Truro, United Kingdom, Sarah.Bell@exeter.ac.uk

Zoe MURRAY, School of Medicine and Menzies Health Institute Queensland, Griffith University, Gold Coast, Australia, z.murray@griffith.edu.au

Anne ROIKO, School of Medicine and Menzies Health Institute Queensland, Griffith University, Gold Coast, Australia, a.roiko@griffith.edu.au
Abstract
A growing body of research demonstrates associations between nature connection and a wide variety of positive health and wellbeing outcomes. Yet, the interpretation of this research is restricted because underpinning mechanisms - particularly the psychological mechanisms of wellbeing enhancement as opposed to wellbeing restoration - remain largely unexplored. Understanding such mechanisms is important for theory development and for assisting 
policy-makers and urban planners to translate this theory into practice effectively. This essay examines the limitations in our current understanding of the psychological mechanisms involved in the relationship between nature connection and eudaimonic wellbeing. It also advances opportunities to move the field forward through exploring two potential mechanisms, namely satisfying the psychological need of relatedness and fostering intrinsic value orientation. These mechanisms may explain how an individual's level of nature connection enhances their psychological wellbeing. Understanding such mechanisms could improve the implementation of targeted nature connection policies and interventions designed to enhance psychological wellbeing among complex urban populations with diverse needs.

\section{Introduction}

Nature exposure has been associated with significant physiological and psychological health benefits. Positive associations have been identified between presence of nature in the living environment and self-reported health (De Vries, Verheij, Groenewegen, \& Spreeuwenberg, 2003; Van den Berg, Maas, Verheij, \& Groenewegen, 2010), as well as reduced morbidity, mortality, stress, obesity and cardiovascular and respiratory disease (James, Hart, Banay, \& Laden, 2016; Maas et al., 2009; Nielsen \& Hansen, 2007; E. Richardson, Pearce, Mitchell, Day, \& Kingham, 2010). Evidence is starting to elucidate a variety of pathways through which such associations might arise. Indeed, several pathway frameworks have been proposed in the literature, with Hartig's four pathways of stress, air quality, physical activity and social contacts being the most commonly cited (Hartig, Mitchell, De Vries, \& Frumkin, 2014). Other frameworks have expanded on this, with one particular framework identifying up to 21 plausible pathways while proposing enhanced immune function as the potential central pathway through which nature delivers multiple health benefits (M. Kuo, 2015). However, such frameworks fail to distinctly link these identified pathways with specific types 
of nature exposures within varying nature settings. Hence, our understanding of how to target specific pathways through the design and delivery of different types of nature exposures remains limited.

What is known, however, is that some pathways are direct and potentially involuntary, such as direct physiological restoration from stress (Ulrich et al., 1991), buffering of anthropogenic noise (Gidlöf-Gunnarsson \& Öhrström, 2010; Pathak, Tripathi, \& Mishra, 2008) and production of natural sounds (Galbrun \& Ali, 2013), reduced urban heat island effect (Loughner et al., 2012), exposure to enhanced air quality (Nowak, Crane, \& Stevens, 2006), airborne phytoncides and negative ions (Craig, Logan, \& Prescott, 2016), ultra violet light which generates vitamin D (Grant \& Holick, 2005), and biologically diverse macro and microbiota that improves the human microbiota (von Hertzen et al., 2015). Other pathways are likely to be indirect and occur through facilitating behavioural and cognitive processes, for example, through providing opportunities for physical activity (Bowler, Buyung-Ali, Knight, \& Pullin, 2010), social interaction (Coley, Sullivan, \& Kuo, 1997; F. E. Kuo, 2003), positive emotional and/or spiritual experiences (Warber, Irvine, Devine-Wright, \& Gaston, 2013), as well as allowing recovery from cognitive fatigue (Kaplan, 1995), reducing antisocial behaviour, particularly in adolescents (Younan et al., 2016) and enhancing perceived community cohesion (Weinstein et al., 2015).

While the majority of literature reports on positive associations between nature exposure and health and wellbeing, more attention needs to be paid to the characteristics of these relationships. A linear assumption underlies most of the literature where an increase in nature exposure is assumed to result in improved health and wellbeing outcomes. However, this relationship will likely be determined by a number of factors such as the type of pathway being examined, the type of nature exposure taking place, the type of nature setting in which 
it occurs and the type of person receiving the exposure. For example, longitudinal research has shown that the association between nature and mental health varies across both the life course and gender with men seeming to derive the greatest benefit of nature exposure during early to mid-adulthood (Astell-Burt, Mitchell, \& Hartig, 2014). Similarly, certain pathways will have a more defined dose-response relationship than others as is the case with ultra violet light exposure, where exceeding a certain dose or exposure can lead to adverse health outcomes (Grant \& Holick, 2005).

The increasing prevalence of mental illness highlights the need to better understand the complex psychological pathways and mechanisms by which nature can promote a sense of wellbeing. Mental and substance use disorders were the leading cause of years lived with disability (YLDs) worldwide in 2010, accounting for 22.9\% of all YLDs (Whiteford et al., 2013). Depression alone accounts for $4.3 \%$ of the global burden of disease and is among the largest single causes of disability worldwide (11\% of all YLDs globally), particularly for women (WHO, 2013). In Australia it is estimated that, over a lifetime, nearly half of the adult population will experience mental illness at some point - equating to nearly 7.3 million Australians aged 16 to 85 (ABS, 2008). Occurrence of mental illness also varies across different sub-groups within a population. Using an Australian example again, adult Indigenous Australians are nearly three times as likely as non-Indigenous adults to experience high to very high levels of psychological distress (ABS, 2013).

Despite the pressing need to combat mental illness and the mounting evidence for the link between nature and wellbeing, currently, little is known about how nature promotes psychological wellbeing. Our understanding of the psychological pathways and mechanisms by which nature can influence mental health and wellbeing is strongest in relation to the recovery of stress and mental ailments (e.g. Ulrich's 1991 Stress Recovery Theory and 
Kaplan's 1995 Attention Restoration Theory). In contrast, our understanding of how nature promotes and sustains psychological wellbeing is much less developed. This is a missed opportunity particularly since many health plans and policies now include the aim of delivering health systems that support contributing and flourishing communities with high psychological wellbeing (NMHC, 2014). Developing a comprehensive understanding of the psychological wellbeing promotion potential of nature in its diverse forms is therefore imperative and forms the main focus of this essay.

\subsection{What do we mean by 'nature'?}

'Nature' is a broad and sometimes ambiguous term. It has a wide variety of objective referents, but is experienced subjectively, with some framing it as a social construction (Castree, 2013; Proctor, 1998). Greider and Garkovich (1994, p.1) describe natural landscapes as "symbolic environments created by human acts of conferring meaning to nature and the environment, of giving the environment definition and form from a particular angle of vision and through a special filter of values and beliefs". Indeed, the meaning and interpretation that people place on nature or natural landscapes can even present contradictions with one study of American adults showing that even people who viewed themselves as part of nature still understood natural landscapes to be those which were free of human interference (Vining, Merrick, \& Price, 2008).'Nature’ is therefore interpreted in diverse ways and has sparked debate in the literature (Hartig et al., 2014). This essay discusses nature from two perspectives, first by presenting an overview of research examining the benefits of exposure to nature and then by introducing the concept of nature connection. Nature exposures are diverse, with multiple variables interacting to create the exposure. According to Frumkin (2013, p. 197) it can extend "from flowers (as in horticultural therapy) to healing gardens, from viewing trees to wilderness adventures, from bird-watching to visiting zoos to owning pets". Within 30 years, $70 \%$ of people will live in 
urban areas (Dye, 2008) and it is therefore the everyday urban nature that will increasingly be where people's nature exposures occur (Dunn, Gavin, Sanchez, \& Solomon, 2006). Hence, this essay is focused primarily on the diverse types of nature exposure accessible within an urban landscape and considers both the 'green' and 'blue' space elements of this urban nature.

Urban nature spans a continuum of different levels of human intervention, design and management, for example from gardens to parks to urban forests, and from canals to rivers to coasts. Regardless of the level of human influence, what is (or isn't) considered to be urban nature will depend on how people perceive the 'naturalness' of the urban nature, with some people valuing elements of urban nature that others disregard as inferior or even 'inauthentic' compared to that encountered in, for example, protected national parks or wilderness areas (Tuan, 1971). The degree to which such settings are perceived as 'urban nature' may depend on people's personal experiences as well as the prevailing cultural representations of nature that they are regularly exposed to (Castree, 2013). Consideration of such personal and cultural conceptions of nature is critical when seeking to define and understand urban nature and the everyday exposures of urban residents. Nature connection, on the other hand, describes the personal mix of feelings, emotions and attitudes that a person has towards nature. We return to the concept of nature connection and elaborate on it in more detail below.

\subsection{What do we mean by 'psychological wellbeing'?}

Despite widespread policy and research interest in understanding and promoting wellbeing, there is little consensus on how to define this concept (Trigwell, Francis, \& Bagot, 2014). Two general perspectives tend to be used to describe wellbeing within the literature: hedonism (also termed subjective wellbeing) and eudaimonism (also termed psychological 
wellbeing) (McMahan \& Estes, 2011; R. M. Ryan \& Deci, 2001). From the hedonic perspective wellbeing relates to happiness, generally defined as the absence of negative affect and the presence of positive affect. Eudaimonism, on the other hand, focuses on prime psychological functioning, self-realization and living life in a full and purposeful way (Deci \& Ryan, 2008). Debate about eudaimonic wellbeing and what exactly it means to have prime psychological functioning has resulted in a number of different conceptualisations. For example, Ryff and Keyes (1995) conceptualize psychological wellbeing as consisting of six facets, including personal growth, self-acceptance, life purpose, mastery, autonomy and positive relatedness. R. M. Ryan and Deci (2000), however, emphasize only competence, autonomy, and relatedness as the important psychological needs, and still others suggest living a purposeful life to be the key factor in psychological wellbeing (Seligman, 2004). Given the growing demand for health plans and policies to deliver thriving and flourishing communities, this essay focuses specifically on eudaimonic wellbeing and the role that urban nature can play in supporting communities with positive psychological functioning. However, it is recognised that a holistic understanding of wellbeing requires attention to the multiple facets of wellbeing, as described above.

This essay explores what is known about the effect of urban nature on psychological (eudaimonic) wellbeing, looking first at nature exposure before building the case for investigating the influence of nature connection on psychological wellbeing specifically. We discuss how our limited understanding of the mechanisms underpinning the nature connection-eudaimonia relationship is impeding effective, practical application of naturehealth research for the delivery of urban landscapes that nurture and promote the psychological wellbeing of urban communities. We examine two potential mechanisms, namely promoting non-human relatedness and fostering intrinsic value orientations, both of which are rooted within Self Determination Theory. Exploring these mechanisms could help 
to better theorise the relationship between eudaimonic wellbeing and nature connection. The concluding section explores future research opportunities that could strengthen our understanding of the mechanisms driving the nature connection-eudaimonia relationship which would help reveal the salutary potential (and limits) of urban nature.

\section{What do we know about the links between eudaimonic wellbeing and nature exposure?}

Nature exposure is shown to consistently affect mental health. While nature exposures can have adverse impacts on mental wellbeing through promoting feelings of fear (C. Milligan \& Bingley, 2007) and anxiety (Skår, 2010), the majority of research reports positive associations (Mantler \& Logan, 2015). However, many of the experimental studies reporting positive mental wellbeing associations are limited by their focus on short-term restorative benefits of single nature exposures (Hartig et al., 2014). Moreover, the nature exposures under examination tend to be artificial or controlled forms of nature exposure usually delivered via virtual means (e.g. nature videos, photos) (Van den Berg, Koole, \& Van der Wulp, 2003). Hence, genuine (rather than scenario-based) feelings of risk, safety concerns or discomfort tend to be removed from such exposures. The direction of association might be quite different if the exposure was administered in situ, in riskier nature, with people who have little experience or familiarity in negotiating such settings. That said, despite such limitations, the growing evidence for the beneficial effects of nature exposure on psychological wellbeing is promising.

To date there have been relatively few experimental studies that have addressed the question of why nature exposure produces beneficial effects for psychological wellbeing, and even fewer studies have explored the potential mechanisms through which the relationship occurs (Mayer, Frantz, Bruehlman-Senecal, \& Dolliver, 2008). Attention Restoration Theory 
(Kaplan, 1995) and Stress Recovery Theory (Ulrich et al., 1991) seek to explain the psychoneuro-endocrine mechanisms through which the restorative and recovery functions of nature exposure occur. Most studies that have explored these theories have tended to concentrate on the effect of nature exposure on short-term physiological and psychological mood states rather than longer-term eudaimonic wellbeing outcomes (Hartig et al., 2014). In addition, Attention Restoration Theory and Stress Recovery Theory both suggest that the benefits of nature exposure are dependent upon the recipient being in various degrees of mental illhealth, whether through cognitive fatigue, or one-off/cumulative episodes of stress. The pathways by which people without chronic or acute mental ailments derive psychological wellbeing benefits through everyday nature exposure are currently under-theorised. This is a pertinent gap in our understanding and inhibits efforts to harness the wellbeing potential of nature exposure by those charged with the promotion of thriving and flourishing communities. In order to address this theoretical lacuna and explore critically the mechanisms underpinning the eudaimonic wellbeing promotion outcomes of nature exposure, we must first consider humankind's relationship with nature. There are various constructs that contribute to our understanding of complex human-nature relationships. These include Biophilia (Wilson, 1984), sense of place (Lewicka, 2011), solastalgia (Albrecht, 2006; Warsini, Mills, \& Usher, 2014), and therapeutic landscapes (Bell, Phoenix, Lovell, \& Wheeler, 2015; Gesler, 1992).

\subsection{The Biophilia Hypothesis}

The Biophilia Hypothesis, popularised by Wilson (1984), proposes that humankind, as part of our species' evolutionary heritage, has an innate need to be around other living things and that we are naturally drawn to those places that, in our pre-historic past, have best facilitated survival, in particular, savannah-type landscapes providing shelter and sustenance (Kellert \& 
Wilson, 1995; Wilson, 1984). The Biophilia Hypothesis suggests that the human-nature relationship is driven by biological evolution. While Kellert (2012) has refined this to include nine underpinning biological values, there is limited empirical evidence to support the Biophilia Hypothesis (Joye \& De Block, 2011; Joye \& Van den Berg, 2011) or to further our understanding of whether innate or learned cultural mechanisms are likely to underpin nature's psychological wellbeing benefits.

\subsection{Sense of place}

A well-established body of multi-disciplinary research devoted to people-place relationships has emerged which examines why people come to develop a 'sense of place' as settings accrue meaning over time. Sense of place is interpreted as a broad construct consisting of three dimensions: (a) place attachment, describing an individual's emotional attachment to a setting, linked to feelings of belonging and rootedness; (b) place identity, reflecting a cognitive connection to a setting or type of setting that provides opportunities to both express and affirm personally salient values and identities; and (c) place dependence, often used to explain a more functional reliance on a specific setting to carry out desired activities and realise the achievement of goals (Kyle, Mowen, \& Tarrant, 2004). Despite a growing body of work examining sense of place (or one or more of its constituent dimensions) in the context of everyday green spaces (Arnberger \& Eder, 2012; Bernardini \& Irvine, 2007; R. L. Ryan, 2005), relatively few studies have examined the complex relationships between sense of place, eudaimonic wellbeing and nature exposure (Scannell, 2013; Scannell \& Gifford, 2016).

One as yet under-researched link between urban nature exposure, place attachment and eudaimonic wellbeing concerns the potential role of urban nature in promoting feelings of comfort, belonging and community attachment amongst regular users (Arnberger \& Eder, 
2012; Rishbeth \& Powell, 2013). Such feelings may, in turn, nurture a sense of individual stability, familiarity and security; factors noted by Manzo (2008) as 'critical ingredients' in psychological wellbeing. Studies examining associations between place attachment and wellbeing suggest that place attachment can affect people's sense of life purpose through providing a central place of significance through which the rest of the world becomes coherent (Casakin \& Kreitler, 2008). Moreover, more recent work is beginning to identify a role for nature in shaping people's favourite or 'most attached' places and the subsequent benefits derived (Scannell, 2013).

There may also be value in examining relationships between urban nature exposure, place identity and eudaimonic wellbeing. Place identity is commonly discussed in relation to four principles: distinctiveness (the desire to differentiate oneself from others); self-esteem (an individual's feeling of self-worth or social value); self-efficacy (a measure of individual capacity or agency); and continuity (the desire to preserve continuity of self-concept over time) (Twigger-Ross \& Uzzell, 1996). The maintenance of a link with a specific place (e.g. a favourite local urban park) can offer individuals a sense of purpose and continuity to their identity, using those places as 'referents to past selves and actions' (Twigger-Ross \& Uzzell, 1996, p.207); this is place referent continuity. Alternatively, self-identity may also be preserved through place congruent continuity; making a conscious effort to seek out places with shared characteristics which seem to represent and allow the expression of personal values, thereby preserving continuity of self as a certain type of person. However, to date, limited effort has been made to examine the links between place identity, eudaimonic wellbeing and nature exposure.

Importantly, studies have also illustrated how place attachment can lead to negative impacts on eudaimonic wellbeing. For example, psychological health can be negatively affected when 
the environment that the person feels an emotional attachment or connection to becomes degraded or lost. Solastalgia describes the distress felt by people who, due to environmental degradation, experience loss of solace and place attachment toward their 'home' environments (Albrecht et al., 2007). Solastalgia is one of the key elements within the environmental distress scale which measures the bio-psycho-social cost of ecosystem disturbance (Higginbotham, Connor, Albrecht, Freeman, \& Agho, 2006). Although not explicitly examined in the context of solastalgia, the distinction between place congruent and place referent continuity may be important in shaping such distress. For example, drawing on the findings of a survey with users of a degraded urban park in Michigan, R. L. Ryan (2005) identified greater levels of distress and personal feelings of loss amongst neighbours and recreational users who expressed a place-specific attachment to that particular urban park, than amongst park staff and volunteers who expressed a more conceptual attachment to that type of urban nature-based setting and responded by seeking out an alternative urban park in which to channel their volunteering efforts. This may reflect the different types of place bonds formed by these urban park users; engaging in environmental improvement efforts within the park may have enhanced volunteers' self-identity as environmentally conscious individuals (suggesting a role for place identity), whereas neighbours and recreational users may have relied on the park more for its unique views, facilities, and building memories over time (reflecting greater place attachment and place dependence). From a health risk perspective, such findings provide valuable insight into humankind's relationship with nature but are derived from a focus on the mental-illness as opposed to the mental-wellbeing aspect of this relationship. 


\subsection{Therapeutic landscapes}

Closely aligned with the sense of place literature (Eyles \& Williams, 2008) is the concept of therapeutic landscapes, used to describe place encounters where "the physical and built environments, social conditions and human perceptions combine to produce an atmosphere which is conducive to healing" (Gesler, 1996, p. 96). Whilst early therapeutic landscape studies focused on ill-being and 'extraordinary' places of healing, such as pilgrimage sites and spas (Gesler, 1992, 1993, 1996), researchers have increasingly turned their attention to people's use of everyday settings to promote and maintain a sense of wellbeing on a routine basis. These settings include, for example, public urban parks, community gardens, riverside and coastal settings (Bell et al., 2015; Christine Milligan, Gatrell, \& Bingley, 2004; Völker \& Kistemann, 2013) and indoor nature exposures (McSweeney, Rainham, Johnson, Sherry, \& Singleton, 2014). The concept of therapeutic landscapes therefore seeks to understand how the physical (both natural and built) attributes of an environment, coupled with the more subjective (and inter-subjective) ways in which people relate to an environment (also shaped by prevailing symbolic and cultural/social interpretations), can combine to determine the degree to which environments are experienced as healing, or health promoting for different people (Gesler, 2003). Despite established understandings of these four dimensions of therapeutic landscapes (natural, built, symbolic and social), the mechanisms by which these four dimensions work in combination to promote health, as well as the contested nature of different 'therapeutic' settings amongst different groups and individuals, remains as of yet, somewhat unclear (Bell et al., 2015).

The human-nature relationship has been investigated from many different perspectives. Whilst drawing on contrasting underpinning explanations (ranging from evolutionary to social) the Biophilia Hypothesis, sense of place, solastalgia and therapeutic landscapes 
concepts deepen our understanding of possible human-nature relationships through highlighting the importance of humankind's co-evolution with(in) nature, the perceived therapeutic and healing qualities of nature and the positive and negative emotional and/or cognitive connections between a person and a location. These are all valuable considerations when seeking to discern the nature-eudaimonia relationship. However, despite these contributions to our understanding, the field still lacks a comprehensive understanding of the mechanisms through which nature promotes eudaimonic wellbeing, and how this varies for different people and via diverse modes of nature exposure.

\section{What do we know about the links between eudaimonic wellbeing and nature connection?}

The gap in our understanding of nature's ability to promote eudaimonic wellbeing amongst different individuals may be addressed by considering an individual's level of nature connection. There is a growing call for researchers, in the nature-health field, to assess what some authors term 'individual agency' when investigating how people engage with nature and the health outcomes associated with that nature exposure (Bell, Phoenix, Lovell, \& Wheeler, 2014). A key aspect of individual agency is a person's self-identification with nature or level of nature connection. In its broadest sense, nature connection describes the mix of feelings, attitudes, beliefs and behaviours that people have towards nature. A range of validated scales exist for measuring an individual's level of nature connection, with the most commonly used scales being the single item 'Inclusion of Nature in Self Scale' (Schultz, 2001), the more recent 'Nature Relatedness Scale' (Nisbet, Zelenski, \& Murphy, 2011) and the Mayer and Frantz (2004) 'Connection to Nature Scale', which has also been adapted to measure nature connection among children (Bragg, Wood, Barton, \& Pretty, 2013). Nature connection scales tend to measure, to varying degrees, the affective (feelings and attitudes), 
cognitive (beliefs and knowledge) and behavioural (actions and experience) aspects of the human-nature connection. The affective domain of this relationship is the most commonly assessed and is reported to be measured by all three of the above mentioned scales, although this is debated in the literature, particularly for the 'Connection to Nature Scale' (Perrin \& Benassi, 2009). Of the three scales, the 'Nature Relatedness Scale' is the only one designed to measure the behavioural domain of the relationship. Higher levels of nature connection have been associated with greater subjective wellbeing (Nisbet et al., 2011) as well as a range of hedonic measures of wellbeing such as vitality, life satisfaction and positive affect (Capaldi, Dopko, \& Zelenski, 2014). While research on nature connection tends to still rely strongly on reported associations with these hedonic measures of wellbeing, there are some promising findings emerging around positive associations between nature connection and eudaimonic measures of wellbeing as measured by the 'Connection to Nature Scale' (Howell, Dopko, Passmore, \& Buro, 2011), the 'Nature Relatedness Scale' and the 'Inclusion of Nature in Self Scale' (Zelenski \& Nisbet, 2014). In addition, higher immersion in nature, as achieved through viewing nature images with guiding audio, predicted high levels of nature connection, as measured using an adapted version of 'Connection to Nature Scale'. This, in turn, predicted greater intrinsic value orientation, which is also linked to eudaimonic wellbeing (Weinstein, Przybylski, \& Ryan, 2009).

'Nature Relatedness' and 'Inclusion of Nature in Self' have been shown to correlate with eudaimonic wellbeing indicators, as measured by the Psychological Well-Being Inventory, but not with ill-being indicators, as measured by the Center for Epidemiological Studies Depression Scale and Negative Affect scale, suggesting that nature connection may play a more useful role in promoting eudaimonic wellbeing as opposed to restoring people from states of ill-health (Zelenski \& Nisbet, 2014). Furthermore, mediational analyses have indicated that the positive health effects of exposure to nature are partially mediated by 
increases in an individual's level of nature connection (Mayer et al., 2008). While the wellbeing promotion potential of nature connection holds promise, it must be noted that the nature connection evidence base tends to rely heavily upon student samples (contributing a third of samples for a recent nature connection meta-analysis) with a strong female bias (65\% median in the same meta-analysis) (Capaldi et al., 2014). In addition, the evidence base is derived mainly from westernised cultures with few studies measuring and assessing the role of nature connection among other cultures. The nature connection studies that do look at nonwesternised cultures tend to also rely on student samples, for example, measuring nature connection among Hong Kong university students using a variety of scales (Tam, 2013).

The growing evidence of nature connection's association with eudaimonic wellbeing and positive health outcomes is coupled with a recent call to action by Craig et al. (2016) for fellow researchers across all disciplines to incorporate validated nature connection scales into diverse research protocols. This would provide critical unifying information from across multiple disciplines and sectors, building the case for the potential importance of nature connection's role in health and wellbeing. However, given that the majority of nature connection scales have been validated within western cultures, there may be need for such scales to be tailored to better resonate with the perceptions and understandings of nature connection among people from diverse and varying cultures.

Exploring an individual's nature connection may be central to unlocking our understanding of how urban nature promotes eudaimonic wellbeing. However, despite the growing interest and accumulating research around the concept of nature connection, there remains limited indepth theoretical work that explores the mechanisms by which a high level of nature connection could promote psychological wellbeing. Moreover, we know little about how this connection manifests itself in relation to varied nature-based settings, in the context of 
different modes of nature exposure, or amongst diverse urban population groups. To date research has instead tended to focus more on understanding nature exposure. Substantial work has been carried out to discern the mediators and moderators influencing the pathways between direct nature exposure and resulting wellbeing outcomes (de Vries, van Dillen, Groenewegen, \& Spreeuwenberg, 2013; Shanahan et al., 2015) but this tends to overlook the different and often highly personal ways in which people conceptualise, value and connect to (or indeed, disconnect from) varied forms of urban nature. Lachowycz and Jones (2013) developed a theoretical framework highlighting the mediators and moderators that drive associations between nature exposure and both physical and psychological health outcomes. The framework proposes three broad groups of mediators (perception of living environment, viewing nature and using nature) and outlines a range of moderators including demographics, living context, nature characteristics and climate. Although this framework is relatively comprehensive, incorporating a number of relevant cultural and socio-economic factors, it does not consider nature connection. Hence, despite the compelling evidence accumulating around nature connection and health, particularly psychological wellbeing, nature connection is still proving elusive when it comes to its incorporation into theory and explanatory frameworks in the nature-health field.

\section{How a limited understanding of mechanisms impedes cultivation of nature connection}

As mentioned above, nature connection is likely to play an important role in promoting eudaimonic wellbeing amongst certain individuals (Capaldi et al., 2014; Capaldi, Passmore, Nisbet, Zelenski, \& Dopko, 2015; Nisbet et al., 2011; Zelenski \& Nisbet, 2014). Hence, there is growing interest in the cultivation of nature connection amongst diverse urban communities. Research indicates that individuals feel closer and more connected to natural 
landscapes after contact with such settings (Mayer et al., 2008; Weinstein et al., 2009). This has catalysed calls for nature exposure to be increased as a cost-effective way to enhance people's nature connection and associated sense of wellbeing (Capaldi et al., 2015; Trigwell et al., 2014). Consequently, a plethora of nature exposure interventions are now commonplace in many countries. For example, the Canadian Mental Health Association's Mood Walks initiative, David Suzuki Foundation's 30x30 Nature Challenge, Australian Natureplay programmes and the United Kingdom's Wild Network and 30 Days Wild campaign led by The Wildlife Trusts, to name a few. In addition, nature exposure interventions can be found increasingly in mental health and addiction literature and practice, sometimes under the guise of 'Green Care'. Green Care interventions, such as group nature walks, have been found to be effective treatments for people with significant mental ill-health (Korpela, Stengård, \& Jussila, 2016; Marselle, Irvine, \& Warber, 2014). Notably, Cutcliffe and Travale (2016) highlight the cultivation of nature connection as critical to the delivery of wellbeing benefits from Green Care.

Despite the growing popularity of these nature exposure interventions, to date only a few have been evaluated to assess their effectiveness for enhancing nature connection. For example, the 30 Days Wild Campaign has been shown to deliver sustained nature connection improvements (M. Richardson, Cormack, McRobert, \& Underhill, 2016). However, the types of nature exposures that occurred during this campaign were not recorded and the authors recognised that those completing all three time points of data collection may have been motivated by a greater nature connection to begin with, therefore potentially clouding our understanding of the effectiveness of such initiatives among general urban communities. Hence, enhancing nature connection and associated wellbeing outcomes through such nature exposure initiatives requires careful consideration of all the factors that constitute the nature exposure. This could include consideration of both the type of nature and type of exposure as 
well as the individual undergoing the exposure and the mediators and moderators that may be at play; we explore these further in what follows.

The types of experiences that occur during the nature exposure will likely influence the degree to which nature connection and diverse wellbeing outcomes can be realised. Some authors propose that there are certain requirements of the nature exposure in order for nature connection to be cultivated and the associated eudaimonic wellbeing outcomes achieved. For example, mindfulness (awareness of the present moment and its associated thoughts, emotions and sensations) and the ability to perceive nature's 'beauty', as measured by the engagement with natural beauty scale, appear to be requirements for achieving maximal connection and health benefits associated with nature exposure across western and Asian cultures (Howell et al., 2011; Howell, Passmore, \& Buro, 2013; Lin, Tsai, Sullivan, Chang, \& Chang, 2014; M. Richardson \& Hallam, 2013; Zhang, Howell, \& Iyer, 2014; Zhang, Piff, Iyer, Koleva, \& Keltner, 2014). Nature exposure may not fulfil its wellbeing enhancement potential if individuals are distracted from mindful awareness and thereby less able to develop a sense of nature connection (Mantler \& Logan, 2015). Conversely, M. Richardson and Sheffield (2015), propose that intentional, reflective self-attention is critical during a nature exposure, with those who are more reflective and inclined to analyse their 'self' indicating a greater increase in nature connection.

The integral role of the individual within this relationship must also be considered. The success of efforts to promote nature connection through increasing nature exposure will be dependent upon the priorities, perceptions, preferences and experiences of the individual under-going the nature exposure. The exposure will be influenced by the individual's unique identities, their current personal projects, past experiences and situational influences (Patterson, Williams, Watson, \& Roggenbuck, 1998); this suggests that people's nature 
exposures and potential nature connection outcomes are idiosyncratic, dynamic and vary across the life course. Similarly, Rose (2012) describes how people's 'prior familiarity' with a landscape or type of landscape can influence the wellbeing outcomes experienced within that landscape, with more familiar landscape types producing greater wellbeing outcomes. Prior familiarity also affects people's landscape preferences as shown in a Swedish study which demonstrated that adults prefer landscape types experienced during childhood (Adevi \& Grahn, 2012). Hence, when designing and delivering urban nature exposures, the nature preferences and previous experiences of local urban residents need to be considered to ensure benefits are delivered and adverse outcomes avoided.

Moderators of the nature connection-eudaimonia relationship have been identified. While the Biophilia Hypothesis (Wilson, 1984) and evolutionary psychology (Barkow, Cosmides, \& Tooby, 1995) propose an evolutionary tendency amongst humans to connect with nature, empirical evidence for such tendencies is limited, and there is therefore a need to better understand the role of developmental experiences and socio-cultural contexts. Cultural differences and prevailing social constructions of nature may play an important role in how people conceptualise their relationship with nature, hence moderating the nature connectioneudaimonia relationship. For example, researchers have observed that some groups (e.g. Menominee Native Americans) are more likely to view themselves as connected to nature compared to other groups (e.g. European Americans), even at relatively early stages in the life course (Bang, Medin, \& Atran, 2007; Unsworth et al., 2012). Furthermore, studies have found that connections with the land over many generations render Indigenous peoples particularly sensitive to the psychological wellbeing outcomes from nature connection (Townsend, Henderson-Wilson, Warner, \& Weiss, 2015). Therefore, nature connection and subsequent wellbeing outcomes are moderated by the diverse conceptualisations of nature connection fostered within varying cultural and social contexts. 
In addition to these moderators, 'purposeful living' and 'spirituality' have been identified as potentially important mediators of the nature connection-eudaimonia relationship. Spirituality can be defined as a person's inner belief system that can serve as a unifying force experienced through one's connecting and becoming (Burkhardt, 1989). Saroglou, Buxant, and Tilquin (2008) found that spirituality is significantly associated with nature connection. Building on this and drawing on 'ecological self' theory, Trigwell et al. (2014) showed that non-religious spirituality emerged as a mediator explaining associations between nature connection and five aspects of eudaimonic wellbeing: autonomy, life purpose, personal growth, self-acceptance and positive relatedness. This extends similar work by Kamitsis and Francis (2013) who also proposed spirituality as a potential mediator explaining how nature connection links to psychological wellbeing, and is complemented by recent work identifying associations between spirituality and nature connection among Pacific Islanders (Nunn et al., 2016). Howell et al. (2011) suggest that another mediator influencing pathways between eudaimonia and nature connection might be purpose in life, again a key dimension of eudaimonic wellbeing. Howell suggests that people who experience a high level of connection to nature gain a sense of meaningful existence, which may in turn boost eudaimonic wellbeing. There is therefore evidence that the nature connection-eudaimonia relationship can be mediated by spirituality and purposeful existence. However, we lack clear understanding of the intricacies and underpinning mechanisms that drive these relationships. By what mechanism does having a high level of nature connection promote a sense of purpose or enhance spirituality? This gap in our understanding limits our ability to design and implement targeted urban nature interventions that deliver on wellbeing outcomes through cultivating nature connection. 


\section{Identifying nature connection mechanisms}

The question of how best to enhance nature connection among urban residents is a difficult one to address. Given the multiple factors at play during a nature exposure, it is not possible to design or prescribe a 'one-size-fits-all' urban nature exposure that will effectively increase nature connection and associated wellbeing outcomes among urban residents, particularly when so little is known about the mechanisms by which nature connection enhances psychological wellbeing. We need to focus research effort initially on identifying the full range of contributing mechanisms, before then discerning how the effectiveness of these mechanisms varies across different conceptions of nature, varied modes of nature exposure (including media modes such as film, sounds and images) and the different cultural and social contexts in which the exposure occurs. This fine-grained understanding will inform the design and delivery of urban nature spaces aimed at enhancing nature connection among complex urban populations with diverse preferences and needs.

A key objective of the current essay is therefore to theorise the potential mechanisms underpinning pathways between eudaimonic wellbeing and nature connection and thereby extend the limited research that has been conducted on this issue. Identifying the psychological mechanisms through which eudaimonic wellbeing is associated with nature connection will help to optimise the design and integration of urban green and blue space interventions that enhance nature connection among urban residents, thereby potentially facilitating delivery of maximum wellbeing benefits. Using the theoretical framework of Self Determination Theory (SDT) (R. M. Ryan \& Deci, 2000), two potential mechanisms are presented here. SDT is a macro theory, comprised of six sub-theories, which explains human motivation and personality. SDT concerns people's inherent growth tendencies and innate psychological needs. The first proposed mechanism, based within the SDT sub-theory of 
Basic Psychological Needs Theory, concerns the potential for nature connection to satisfy the psychological need of relatedness. The second mechanism, based within the SDT sub-theory of Goal Contents Theory, explores how nature connection may foster an intrinsic value orientation and associated wellbeing outcomes. We expand on these sub-theories below, and the role they may play in the nature connection-eudaimonia relationship.

\subsection{Promoting non-human relatedness}

Satisfaction of the basic psychological need of relatedness may explain the underlying mechanism through which nature connection affects positive psychological wellbeing. According to the Basic Psychological Needs Theory of SDT, relatedness, along with competence and autonomy, are seen as basic psychological needs. Behaviours and contexts that allow for the experience of relatedness, autonomy and competence, support basic psychological needs and thereby enhance an individual's sense of integrity and eudaimonic wellbeing (R. M. Ryan \& Deci, 2000). The psychological need of relatedness refers to the basic and innate need for all humans to relate and connect to others or to the world around them. To date relatedness has mainly been considered from the perspective of interaction, connection and caring for other people. However, it is recognised that while relatedness needs are often satisfied through interactions with others, they are not necessarily exclusively satisfied in this way (Deci \& Ryan, 2000).

Nature connection appears largely distinct from other types of connection or relatedness. Even after controlling for connections that could satisfy relatedness (e.g. family or culture), Zelenski and Nisbet (2014) found that nature connection, as measured by the 'Nature Relatedness Scale', still significantly predicted happiness. This suggests that nature connection may be a type of relatedness, thereby satisfying this basic psychological need. Shiota, Keltner, and Mossman (2007) found that participants recalling experiences where 
they felt nature's 'beauty' gave higher ratings to statements such as "I felt connected with the world around me." Indeed, for some individuals (e.g. those with high levels of stress or more severe disabilities such as autism), social relationships can prove overwhelming, with comfort gained instead from simple relationships with non-human forms of nature (Davidson \& Smith, 2009; Ottosson, 2001; Ottosson \& Grahn, 2008). Furthering the case for non-human forms of relatedness is the well-established research demonstrating that bonds between humans and animals, particularly pets, can help fulfil relatedness needs (Podberscek, Paul, \& Serpell, 2005). These findings suggest that nature connection may serve as a non-human form of relatedness, hence satisfying the basic psychological need for relatedness and potentially explaining how nature connection promotes eudaimonic wellbeing.

\subsection{Fostering intrinsic value orientations}

The Goals Contents Theory of SDT focuses on value-orientations and aspirations and may explain another potential mechanism through which nature connection influences psychological wellbeing. Specifically, intrinsic aspirations involve the pursuit of goals concerning personal growth, intimacy and community, and have been shown to be associated with greater eudaimonic wellbeing (Kasser et al., 2014; R. M. Ryan, Huta, \& Deci, 2008). Extrinsic aspirations relate to externally valued goods that are not inherently rewarding but are sought to derive positive regard or rewards from others (e.g. money, image, status and fame). Extrinsic aspirations are associated with lower scores for outcomes such as life satisfaction, happiness, vitality, and self-actualization, and higher scores for outcomes such as depression, anxiety, behaviour disorders, and a host of other types of psychopathologies (Kasser, 2003; Kasser \& Ryan, 1993). Nature connection is positively associated with a variety of intrinsic aspirations, including humanitarianism (Nisbet, Zelenski, \& Murphy, 2008), kindness (Leary, Tipsord, \& Tate, 2008), empathic concern (Zhang, Piff, et al., 2014) 
and altruistic concern (Schultz, 2001). Nature connection has also been linked to behaviours indicative of intrinsic aspiration, for example relational emotions (e.g. love and care) (Vining et al., 2008), less selfish consumer decision making (Mayer \& Frantz, 2004) and proenvironmental decision making (Vining, 1987). In addition to nature connection, direct exposure to nature is also associated with intrinsic aspirations. After viewing nature images participants reported higher valuing of intrinsic aspirations and lower valuing of extrinsic aspirations, whereas those who viewed images of urban landscapes, which lacked nature, reported increased valuing of extrinsic aspirations and no change of intrinsic aspirations (Weinstein et al., 2009). Similarly, briefly viewing either unspectacular or awe-evoking photographs of nature can promote people's intrinsic aspirations making them feel more caring, spiritual and connected to others (Joye \& Bolderdijk, 2014). Taken together this research suggests that value orientations and aspirations may be implicated in the relationship between eudaimonic wellbeing and nature connection. On this basis we theorise that nature connection may be related to wellbeing because it helps to increase intrinsic value orientations and aspirations. By increasing nature connection, it is plausible that intrinsic aspirations could be promoted and resulting eudaimonic wellbeing benefits delivered. Further research on nature connection should assess people's value-orientations to ascertain whether (and if so, how and why) eudaimonic wellbeing benefits are delivered through the mechanism of nature connection fostering intrinsic values within people.

\section{Conclusions}

Promoting feelings of eudaimonic wellbeing through forging connections to nature has important consequences for psychological health, particularly among urban residents. This essay builds on previous recommendations to consider individual agency within the naturehealth research field (Bell et al., 2014), incorporate nature connection into study protocols 
(Craig et al., 2016) and to cultivate nature connection among urban communities (Trigwell et al., 2014). It critically examines what is known about the nature connection and eudaimonic wellbeing relationship, exploring the current state of evidence for the potential moderators (e.g. cultural constructions/interpretations of nature) and mediators (e.g. mindfulness, spirituality, purpose in life) that could influence this relationship. In so doing, it identifies pertinent gaps in our understanding of this relationship, namely the need to identify and understand its underpinning mechanisms.

Using Self Determination Theory, we propose two potential mechanisms for further investigation in order to address this gap: satisfaction of the psychological need of relatedness and fostering an intrinsic value orientation. This is not to suggest that these are the only mechanisms that may be at play within the nature connection-eudaimonia relationship, or that their influence will be uniform across different individuals and groups; rather, we have examined the existing literature to identify these two mechanisms as worthy of further investigation. More broadly, this essay seeks to stimulate and contribute to the ongoing debate, among nature and health researchers, on the wellbeing promotion potential of nature connection, and to encourage researchers to include and test the two mechanisms suggested within their study protocols.

By gaining a deeper understanding of such mechanisms, we can better inform the implementation of targeted policies and interventions designed to enhance psychological wellbeing through the cultivation of nature connection among urban populations. Already there are growing calls within the policy arena for increased access to urban green spaces, as with the Sustainable Development Goal 11.7 (UN, 2015), and for urban green space to be part of children's daily routines (WHO, 2010). Similarly, there are recent moves to explore the health promotion potential of 'green prescriptions' that seek to administer a 'healthy dose 
of nature' to target adult populations (Shanahan et al., 2016). However, such interventions that aim to improve health and wellbeing through simply increasing the provision of and/or access to urban nature, in isolation of targeted interventions to connect and engage people with the nature space, may not deliver on the intended health outcomes (Hunter et al., 2015). In order for urban nature to deliver on its reported multiple outcomes the connection and relationship occurring between the nature and the person experiencing it needs to be understood and facilitated within the design and delivery of the urban nature. Certain initiatives already have this relationship as their central focus, for example, widespread initiatives to reconnect children to nature (Louv, 2008) and campaigns such as 30 Days Wild (M. Richardson et al., 2016). However, these initiatives are delivered without an established understanding of the nature connection mechanisms at play. Such understanding could enhance the efficacy of urban nature connection programmes helping to explain how and why different individuals and cultural groups feel able (or otherwise) to connect with diverse forms of nature in their living environments, in what ways, and to what extent (if at all) such experiences enhance feelings of eudaimonic wellbeing. Future research should also explore the role of individual difference factors as influencers on programs aiming to increase wellbeing through nature connection. For example, recent research suggests that agreeableness, conscientiousness and openness to new experiences are the personality traits most strongly linked to environmental engagement, whereas extraversion and neuroticism are less so (Milfont \& Sibley, 2012).

Such insights could help unravel the intricacies involved in designing and delivering effective urban nature connection experiences for diverse urban populations. Should satisfaction of the psychological need of relatedness and fostering an intrinsic value orientation be shown to be the key mechanisms through which nature connection promotes eudaimonic wellbeing, then this understanding could be used by landscape designers and urban planners to inform how 
urban green and blue space interventions are designed and implemented. For example, delivering urban nature spaces that allow people the opportunity to contemplate and reflect on their own relationship and connection with nature, or that actively promote people's sense of connection with nature through facilitating meaningful nature experiences, may promote the feeling of non-human relatedness, hence satisfying this basic psychological need. Urban gardening is showing promise as one way to provide meaningful nature experiences among urban residents (Buck, 2016). Similarly, providing urban nature spaces that enable urban residents to contribute and 'give back' to the community may be a way to promote nature connection and an intrinsic value orientation. Providing conservation and green volunteer opportunities within urban nature spaces may be a possible way to achieve this (Husk, Lovell, Cooper, Stahl- Timmins, \& Garside, 2016). Identifying and understanding the mechanism(s) responsible for the wellbeing outcomes from nature connection will enable interventions to be designed in a way that specifically targets and influences the identified mechanism promoting the desired outcome. This will help move the application of nature and health research beyond 'one-size-fits-all' and 'more-is-better' type interventions towards more tailored, targeted and effective solutions.

Understanding the underpinning mechanisms can inform efforts to promote and/or retrofit 'healthy' green infrastructure networks within and between cities, towns and villages, promoting opportunities for residents to develop their nature connection and wellbeing, as well as offering more widely acknowledged sustainable transport and ecological benefits. 


\section{References}

ABS. (2008). National Survey of Mental Health and Wellbeing 2007: Summary of Results. . Canberra Retrieved from http://www.abs.gov.au/ausstats/abs@.nsf/mf/4326.0.

ABS. (2013). Australian Aboriginal and Torres Strait Islander health survey: first results, Australia, 2012-13: Table 7 [data cube]. Canberra: Australian Bureau of Statistics.

Adevi, A. A., \& Grahn, P. (2012). Preferences for landscapes: A matter of cultural determinants or innate reflexes that point to our evolutionary background? Landscape research, 37(1), 27-49.

Albrecht, G. (2006). Solastalgia. Alternatives Journal, 32(4-5), 34-36.

Albrecht, G., Sartore, G. M., Connor, L., Higginbotham, N., Freeman, S., Kelly, B., . . Pollard, G. (2007). Solastalgia: The distress caused by environmental change. Australasian Psychiatry, 15(SUPPL. 1), S95-S98. doi:10.1080/10398560701701288

Arnberger, A., \& Eder, R. (2012). The influence of green space on community attachment of urban and suburban residents. Urban Forestry \& Urban Greening, 11(1), 41-49. doi:http://dx.doi.org/10.1016/j.ufug.2011.11.003

Astell-Burt, T., Mitchell, R., \& Hartig, T. (2014). The association between green space and mental health varies across the lifecourse. A longitudinal study. Journal of epidemiology and community health, 68(6), 578-583.

Bang, M., Medin, D. L., \& Atran, S. (2007). Cultural mosaics and mental models of nature. Proceedings of the National Academy of Sciences, 104(35), 13868-13874.

Barkow, J. H., Cosmides, L., \& Tooby, J. (1995). The adapted mind: Evolutionary psychology and the generation of culture: Oxford University Press.

Bell, S. L., Phoenix, C., Lovell, R., \& Wheeler, B. W. (2014). Green space, health and wellbeing: Making space for individual agency. Health \& Place, 30, 287-292.

Bell, S. L., Phoenix, C., Lovell, R., \& Wheeler, B. W. (2015). Seeking everyday wellbeing: The coast as a therapeutic landscape. Social science \& medicine, 142, 56-67.

Bernardini, C., \& Irvine, K. (2007). The 'nature' of urban sustainability: private or public greenspaces? WIT Transactions on Ecology and the Environment, 102.

Bowler, D. E., Buyung-Ali, L. M., Knight, T. M., \& Pullin, A. S. (2010). A systematic review of evidence for the added benefits to health of exposure to natural environments. BMC Public Health, 10. doi:10.1186/1471-2458-10-456

Bragg, R., Wood, C., Barton, J., \& Pretty, J. (2013). Measuring connection to nature in children aged 8-12: A robust methodology for the RSPB.

Buck, D. (2016). Gardens and health Implications for policy and practice.

Burkhardt, M. A. (1989). Spirituality: An analysis of the concept. Holistic nursing practice, 3(3), 69-77.

Capaldi, C. A., Dopko, R. L., \& Zelenski, J. M. (2014). The relationship between nature connectedness and happiness: a meta-analysis. Frontiers in Psychology, 5. doi:10.3389/fpsyg.2014.00976

Capaldi, C. A., Passmore, H.-A., Nisbet, E. K., Zelenski, J. M., \& Dopko, R. L. (2015). Flourishing in nature: A review of the benefits of connecting with nature and its application as a wellbeing intervention. International Journal of Wellbeing, 5(4).

Casakin, H. P., \& Kreitler, S. (2008). Place attachment as a function of meaning assignment. Open Environmental Sciences Journal, 2(1).

Castree, N. (2013). Making sense of nature: Routledge.

Coley, R. L., Sullivan, W. C., \& Kuo, F. E. (1997). Where does community grow? The social context created by nature in urban public housing. Environment and Behavior, 29(4), 468-494.

Craig, J. M., Logan, A. C., \& Prescott, S. L. (2016). Natural environments, nature relatedness and the ecological theater: connecting satellites and sequencing to shinrin-yoku. Journal of Physiological Anthropology, 35(1), 1.

Cutcliffe, J. R., \& Travale, R. (2016). Unearthing the Theoretical Underpinnings of "Green Care" in Mental Health and Substance Misuse Care: Theoretical Underpinnings and Contemporary Clinical Examples. Issues in Mental Health Nursing, 1-9. 
Davidson, J., \& Smith, M. (2009). Autistic autobiographies and more-than-human emotional geographies. Environment and Planning D: Society and Space, 27(5), 898-916.

de Vries, S., van Dillen, S. M., Groenewegen, P. P., \& Spreeuwenberg, P. (2013). Streetscape greenery and health: Stress, social cohesion and physical activity as mediators. Social science \& medicine, 94, 26-33.

De Vries, S., Verheij, R. A., Groenewegen, P. P., \& Spreeuwenberg, P. (2003). Natural environmentshealthy environments? An exploratory analysis of the relationship between greenspace and health. Environment and planning A, 35(10), 1717-1732.

Deci, E. L., \& Ryan, R. M. (2000). The" what" and" why" of goal pursuits: Human needs and the selfdetermination of behavior. Psychological inquiry, 11(4), 227-268.

Deci, E. L., \& Ryan, R. M. (2008). Hedonia, eudaimonia, and well-being: An introduction. Journal of Happiness Studies, 9(1), 1-11.

Dunn, R. R., Gavin, M. C., Sanchez, M. C., \& Solomon, J. N. (2006). The pigeon paradox: dependence of global conservation on urban nature. Conservation Biology, 20(6), 1814-1816.

Dye, C. (2008). Health and urban living. Science, 319(5864), 766-769.

Eyles, J., \& Williams, A. (2008). Sense of place, health and quality of life: Ashgate Publishing, Ltd.

Frumkin, H. (2013). The evidence of nature and the nature of evidence. American Journal of Preventive Medicine, 44(2), 196-197.

Galbrun, L., \& Ali, T. T. (2013). Acoustical and perceptual assessment of water sounds and their use over road traffic noisea). The Journal of the Acoustical Society of America, 133(1), 227-237. doi:doi:http://dx.doi.org/10.1121/1.4770242

Gesler, W. M. (1992). Therapeutic landscapes: medical issues in light of the new cultural geography. Social science \& medicine, 34(7), 735-746.

Gesler, W. M. (1993). Therapeutic landscapes: theory and a case study of Epidauros, Greece. Environment and Planning D: Society and Space, 11(2), 171-189.

Gesler, W. M. (1996). Lourdes: healing in a place of pilgrimage. Health \& Place, 2(2), 95-105.

Gesler, W. M. (2003). Healing places: Rowman \& Littlefield Publishers.

Gidlöf-Gunnarsson, A., \& Öhrström, E. (2010). Attractive "Quiet" Courtyards: A Potential Modifier of Urban Residents' Responses to Road Traffic Noise? International Journal of Environmental Research and Public Health, 7(9). doi:10.3390/ijerph7093359

Grant, W. B., \& Holick, M. F. (2005). Benefits and requirements of vitamin D for optimal health: a review. Altern Med Rev, 10(2), 94-111.

Greider, T., \& Garkovich, L. (1994). Landscapes: The social construction of nature and the environment. Rural Sociology, 59(1), 1-24.

Hartig, T., Mitchell, R., De Vries, S., \& Frumkin, H. (2014). Nature and health. Annual Review of Public Health, 35, 207-228.

Higginbotham, N., Connor, L., Albrecht, G., Freeman, S., \& Agho, K. (2006). Validation of an environmental distress scale. EcoHealth, 3(4), 245-254. doi:10.1007/s10393-006-0069-x

Howell, A. J., Dopko, R. L., Passmore, H.-A., \& Buro, K. (2011). Nature connectedness: Associations with well-being and mindfulness. Personality and Individual Differences, 51(2), 166-171. doi:10.1016/j.paid.2011.03.037

Howell, A. J., Passmore, H.-A., \& Buro, K. (2013). Meaning in nature: meaning in life as a mediator of the relationship between nature connectedness and well-being. Journal of Happiness Studies, 14(6), 1681-1696.

Hunter, R. F., Christian, H., Veitch, J., Astell-Burt, T., Hipp, J. A., \& Schipperijn, J. (2015). The impact of interventions to promote physical activity in urban green space: a systematic review and recommendations for future research. Social science \& medicine, 124, 246-256.

Husk, K., Lovell, R., Cooper, C., Stahl-Timmins, W., \& Garside, R. (2016). Participation in environmental enhancement and conservation activities for health and well-being in adults: a review of quantitative and qualitative evidence. The Cochrane Library. 
James, P., Hart, J. E., Banay, R. F., \& Laden, F. (2016). Exposure to Greenness and Mortality in a Nationwide Prospective Cohort Study of Women. Environ Health Perspect.

Joye, Y., \& Bolderdijk, J. W. (2014). An exploratory study into the effects of extraordinary nature on emotions, mood, and prosociality. Frontiers in Psychology, 5.

Joye, Y., \& De Block, A. (2011). 'Nature and I are Two': A Critical Examination of the Biophilia Hypothesis. Environmental Values, 20(2), 189-215.

Joye, Y., \& Van den Berg, A. (2011). Is love for green in our genes? A critical analysis of evolutionary assumptions in restorative environments research. Urban Forestry \& Urban Greening, 10(4), 261-268.

Kamitsis, I., \& Francis, A. J. (2013). Spirituality mediates the relationship between engagement with nature and psychological wellbeing. Journal of Environmental Psychology, 36, 136-143.

Kaplan, S. (1995). The restorative benefits of nature: Toward an integrative framework. Journal of Environmental Psychology, 15(3), 169-182.

Kasser, T. (2003). The high price of materialism: MIT press.

Kasser, T., Rosenblum, K. L., Sameroff, A. J., Deci, E. L., Niemiec, C. P., Ryan, R. M., . . . Dungan, N. (2014). Changes in materialism, changes in psychological well-being: Evidence from three longitudinal studies and an intervention experiment. Motivation and Emotion, 38(1), 1-22.

Kasser, T., \& Ryan, R. M. (1993). A dark side of the American dream: correlates of financial success as a central life aspiration. Journal of personality and social psychology, 65(2), 410.

Kellert, S. R. (2012). Birthright: People and nature in the modern world: Yale University Press.

Kellert, S. R., \& Wilson, E. O. (1995). The biophilia hypothesis: Island Press.

Korpela, K. M., Stengård, E., \& Jussila, P. (2016). Nature Walks as a Part of Therapeutic Intervention for Depression. Ecopsychology, 8(1), 8-15.

Kuo, F. E. (2003). Social aspects of urban forestry: The role of arboriculture in a healthy social ecology.

Kuo, M. (2015). How might contact with nature promote human health? Promising mechanisms and a possible central pathway. Frontiers in Psychology, 6, 1093. doi:10.3389/fpsyg.2015.01093

Kyle, G. T., Mowen, A. J., \& Tarrant, M. (2004). Linking place preferences with place meaning: An examination of the relationship between place motivation and place attachment. Journal of Environmental Psychology, 24(4), 439-454. doi:http://dx.doi.org/10.1016/i.jenvp.2004.11.001

Lachowycz, K., \& Jones, A. P. (2013). Towards a better understanding of the relationship between greenspace and health: development of a theoretical framework. Landscape and Urban Planning, 118, 62-69.

Leary, M. R., Tipsord, J. M., \& Tate, E. B. (2008). Allo-inclusive identity: Incorporating the social and natural worlds into one's sense of self.

Lewicka, M. (2011). Place attachment: How far have we come in the last 40 years? Journal of Environmental Psychology, 31(3), 207-230.

Lin, Y.-H., Tsai, C.-C., Sullivan, W. C., Chang, P.-J., \& Chang, C.-Y. (2014). Does awareness effect the restorative function and perception of street trees. Front Psychol, 5, 906.

Loughner, C. P., Allen, D. J., Zhang, D.-L., Pickering, K. E., Dickerson, R. R., \& Landry, L. (2012). Roles of urban tree canopy and buildings in urban heat island effects: Parameterization and preliminary results. Journal of Applied Meteorology and Climatology, 51(10), 1775-1793.

Louv, R. (2008). Last child in the woods: Saving our children from nature-deficit disorder: Algonquin Books.

Maas, J., Verheij, R. A., de Vries, S., Spreeuwenberg, P., Schellevis, F. G., \& Groenewegen, P. P. (2009). Morbidity is related to a green living environment. Journal of Epidemiology \& Community Health, 63(12), 967-973. doi:10.1136/jech.2008.079038

Mantler, A., \& Logan, A. C. (2015). Natural environments and mental health. Advances in Integrative Medicine. 
Manzo, L. C. (2008). The experience of displacement on sense of place and well-being. Sense of place, health and quality of life, 87104.

Marselle, M. R., Irvine, K. N., \& Warber, S. L. (2014). Examining group walks in nature and multiple aspects of well-being: A large-scale study. Ecopsychology, 6(3), 134-147.

Mayer, F. S., \& Frantz, C. M. (2004). The connectedness to nature scale: A measure of individuals' feeling in community with nature. Journal of environmental psychology, 24(4), 503-515.

Mayer, F. S., Frantz, C. M., Bruehlman-Senecal, E., \& Dolliver, K. (2008). Why is nature beneficial? The role of connectedness to nature. Environment and Behavior.

McMahan, E. A., \& Estes, D. (2011). Hedonic versus eudaimonic conceptions of well-being: Evidence of differential associations with self-reported well-being. Social Indicators Research, 103(1), 93-108.

McSweeney, J., Rainham, D., Johnson, S., Sherry, S., \& Singleton, J. (2014). Indoor nature exposure (INE): a health-promotion framework. Health promotion international, dau081.

Milfont, T. L., \& Sibley, C. G. (2012). The big five personality traits and environmental engagement: Associations at the individual and societal level. Journal of Environmental Psychology, 32(2), 187-195.

Milligan, C., \& Bingley, A. (2007). Restorative places or scary spaces? The impact of woodland on the mental well-being of young adults. Health and Place, 13(4), 799-811. doi:10.1016/j.healthplace.2007.01.005

Milligan, C., Gatrell, A., \& Bingley, A. (2004). 'Cultivating health': therapeutic landscapes and older people in northern England. Social science \& medicine, 58(9), 1781-1793.

Nielsen, T. S., \& Hansen, K. B. (2007). Do green areas affect health? Results from a Danish survey on the use of green areas and health indicators. Health and Place, 13(4), 839-850. doi:10.1016/j.healthplace.2007.02.001

Nisbet, E. K., Zelenski, J. M., \& Murphy, S. A. (2008). The nature relatedness scale: Linking individuals' connection with nature to environmental concern and behavior. Environment and Behavior.

Nisbet, E. K., Zelenski, J. M., \& Murphy, S. A. (2011). Happiness is in our nature: Exploring nature relatedness as a contributor to subjective well-being. Journal of Happiness Studies, 12(2), 303-322.

NMHC. (2014). The National Review of Mental Health Programmes and Services. National Mental Health Commission, Sydney, Australia.

Nowak, D. J., Crane, D. E., \& Stevens, J. C. (2006). Air pollution removal by urban trees and shrubs in the United States. Urban forestry \& urban greening, 4(3), 115-123.

Nunn, P. D., Mulgrew, K., Scott-Parker, B., Hine, D. W., Marks, A. D., Mahar, D., \& Maebuta, J. (2016). Spirituality and attitudes towards Nature in the Pacific Islands: insights for enabling climatechange adaptation. Climatic Change, 1-17.

Ottosson, J. (2001). The Importance of Nature in Coping with a Crisis: A photographic essay. Landscape research, 26(2), 165-172. doi:10.1080/01426390120045436

Ottosson, J., \& Grahn, P. (2008). The role of natural settings in crisis rehabilitation: how does the level of crisis influence the response to experiences of nature with regard to measures of rehabilitation? Landscape research, 33(1), 51-70.

Pathak, V., Tripathi, B. D., \& Mishra, V. K. (2008). Dynamics of traffic noise in a tropical city Varanasi and its abatement through vegetation. Environmental Monitoring and Assessment, 146(1), 67-75. doi:10.1007/s10661-007-0060-1

Patterson, M. E., Williams, D. R., Watson, A. E., \& Roggenbuck, J. R. (1998). An hermeneutic approach to studying the nature of wilderness experiences. Journal of Leisure Research, 30(4), 423.

Perrin, J. L., \& Benassi, V. A. (2009). The connectedness to nature scale: A measure of emotional connection to nature? Journal of Environmental Psychology, 29(4), 434-440.

Podberscek, A. L., Paul, E. S., \& Serpell, J. A. (2005). Companion animals and us: Exploring the relationships between people and pets: Cambridge University Press. 
Proctor, J. D. (1998). The social construction of nature: Relativist accusations, pragmatist and critical realist responses. Annals of the Association of American Geographers, 88(3), 352-376.

Richardson, E., Pearce, J., Mitchell, R., Day, P., \& Kingham, S. (2010). The association between green space and cause-specific mortality in urban New Zealand: An ecological analysis of green space utility. BMC Public Health, 10. doi:10.1186/1471-2458-10-240

Richardson, M., Cormack, A., McRobert, L., \& Underhill, R. (2016). 30 Days Wild: Development and Evaluation of a Large-Scale Nature Engagement Campaign to Improve Well-Being. Plos One, 11(2), e0149777.

Richardson, M., \& Hallam, J. (2013). Exploring the psychological rewards of a familiar semirural landscape: Connecting to local nature through a mindful approach. The Humanistic Psychologist, 41(1), 35-53.

Richardson, M., \& Sheffield, D. (2015). Reflective Self-Attention: A More Stable Predictor of Connection to Nature Than Mindful Attention. Ecopsychology, 7(3), 166-175.

Rishbeth, C., \& Powell, M. (2013). Place attachment and memory: landscapes of belonging as experienced post-migration. Landscape research, 38(2), 160-178.

Rose, E. (2012). Encountering place: a psychoanalytic approach for understanding how therapeutic landscapes benefit health and wellbeing. Health \& Place, 18(6), 1381-1387.

Ryan, R. L. (2005). Exploring the effects of environmental experience on attachment to urban natural areas. Environment and Behavior, 37(1), 3-42.

Ryan, R. M., \& Deci, E. L. (2000). Self-determination theory and the facilitation of intrinsic motivation, social development, and well-being. American Psychologist, 55(1), 68.

Ryan, R. M., \& Deci, E. L. (2001). On happiness and human potentials: A review of research on hedonic and eudaimonic well-being. Annual review of psychology, 52(1), 141-166.

Ryan, R. M., Huta, V., \& Deci, E. L. (2008). Living well: A self-determination theory perspective on eudaimonia. Journal of Happiness Studies, 9(1), 139-170.

Ryff, C. D., \& Keyes, C. L. M. (1995). The structure of psychological well-being revisited. Journal of personality and social psychology, 69(4), 719.

Saroglou, V., Buxant, C., \& Tilquin, J. (2008). Positive emotions as leading to religion and spirituality. The Journal of Positive Psychology, 3:3, $165-173$.

Scannell, L. (2013). The Bases of Bonding: The Psychological Functions of Place Attachment in Comparison to Interpersonal Attachment. (DOCTOR OF PHILOSOPHY), University of Victoria.

Scannell, L., \& Gifford, R. (2016). Place Attachment Enhances Psychological Need Satisfaction. Environment and Behavior. doi:10.1177/0013916516637648

Schultz, W. P. (2001). The Structure of Environmental Concern: Concern for Self, Other People and the Biosphere Journal of Environmental Psychology, 21(4), 327-339. doi:http://dx.doi.org/10.1006/jevp.2001.0227

Seligman, M. E. (2004). Authentic happiness: Using the new positive psychology to realize your potential for lasting fulfillment: Simon and Schuster.

Shanahan, D., Bush, R., Gaston, K., Lin, B., Dean, J., Barber, E., \& Fuller, R. (2016). Health benefits from nature experiences depend on dose. Scientific reports, 6 .

Shanahan, D., Lin, B., Bush, R., Gaston, K., Dean, J., Barber, E., \& Fuller, R. (2015). Toward improved public health outcomes from urban nature. American Journal of Public Health, 105(3), 470477.

Shiota, M. N., Keltner, D., \& Mossman, A. (2007). The nature of awe: Elicitors, appraisals, and effects on self-concept. Cognition and emotion, 21(5), 944-963.

Skår, M. (2010). Forest dear and forest fear: Dwellers' relationships to their neighbourhood forest. Landscape and Urban Planning, 98(2), 110-116.

Tam, K.-P. (2013). Concepts and measures related to connection to nature: Similarities and differences. Journal of Environmental Psychology, 34, 64-78.

Townsend, M., Henderson-Wilson, C., Warner, E., \& Weiss, L. (2015). Healthy Parks Healthy People: the state of the evidence 2015. 
Trigwell, J. L., Francis, A. J., \& Bagot, K. L. (2014). Nature connectedness and eudaimonic well-being: Spirituality as a potential mediator. Ecopsychology, 6(4), 241-251.

Tuan, Y. F. (1971). Geography, phenomenology, and the study of human nature. The Canadian Geographer/Le Géographe canadien, 15(3), 181-192.

Twigger-Ross, C. L., \& Uzzell, D. L. (1996). Place and identity processes. Journal of Environmental Psychology, 16(3), 205-220.

Ulrich, R. S., Simons, R. F., Losito, B. D., Fiorito, E., Miles, M. A., \& Zelson, M. (1991). Stress recovery during exposure to natural and urban environments. Journal of Environmental Psychology, 11(3), 201-230.

UN. (2015). United Nations: Transforming our world: the 2030 Agenda for Sustainable Development Unsworth, S. J., Levin, W., Bang, M., Washinawatok, K., Waxman, S. R., \& Medin, D. L. (2012). Cultural differences in children's ecological reasoning and psychological closeness to nature: Evidence from Menominee and European American children. Journal of Cognition and Culture, 12(1-2), 17-29.

Van den Berg, A. E., Koole, S. L., \& Van der Wulp, N. Y. (2003). Environmental preference and restoration: (How) are they related? Journal of Environmental Psychology, 23(2), 135-146. doi:10.1016/s0272-4944(02)00111-1

Van den Berg, A. E., Maas, J., Verheij, R. A., \& Groenewegen, P. P. (2010). Green space as a buffer between stressful life events and health. Social science \& medicine, 70(8), 1203-1210.

Vining, J. (1987). Environmental decisions: The interaction of emotions, information, and decision context. Journal of Environmental Psychology, 7(1), 13-30.

Vining, J., Merrick, M. S., \& Price, E. A. (2008). The distinction between humans and nature: Human perceptions of connectedness to nature and elements of the natural and unnatural. Human Ecology Review, 15(1), 1.

Völker, S., \& Kistemann, T. (2013). "I'm always entirely happy when I'm here!" Urban blue enhancing human health and well-being in Cologne and Düsseldorf, Germany. Social Science and Medicine, 78(1), 113-124. doi:10.1016/j.socscimed.2012.09.047

von Hertzen, L., Beutler, B., Bienenstock, J., Blaser, M., Cani, P. D., Eriksson, J., . . Jenmalm, M. C. (2015). Helsinki alert of biodiversity and health. Annals of medicine(0), 1-8.

Warber, S., Irvine, K., Devine-Wright, P., \& Gaston, K. (2013). Modeling well-being and the relationship between individuals and their environment: Routledge, New York, NY, USA.

Warsini, S., Mills, J., \& Usher, K. (2014). Solastalgia: Living with the environmental damage caused by natural disasters. Prehospital and Disaster Medicine, 29(1), 87-90. doi:10.1017/S1049023X13009266

Weinstein, N., Balmford, A., DeHaan, C. R., Gladwell, V., Bradbury, R. B., \& Amano, T. (2015). Seeing Community for the Trees: The Links among Contact with Natural Environments, Community Cohesion, and Crime. BioScience, 65(12), 1141-1153. doi:10.1093/biosci/biv151

Weinstein, N., Przybylski, A. K., \& Ryan, R. M. (2009). Can nature make us more caring? Effects of immersion in nature on intrinsic aspirations and generosity. Personality and Social Psychology Bulletin, 35(10), 1315-1329.

Whiteford, H. A., Degenhardt, L., Rehm, J., Baxter, A. J., Ferrari, A. J., Erskine, H. E., . . Johns, N. (2013). Global burden of disease attributable to mental and substance use disorders: findings from the Global Burden of Disease Study 2010. The Lancet, 382(9904), 1575-1586.

WHO. (2010). Parma declaration on Environment and Health. Fifth Ministerial Conference on Environment and Health "Protecting children's health in a changing environment". . Copenhagen: WHO Regional Office for Europe.

WHO. (2013). World Health Organisation Mental health action plan 2013-2020.

Wilson, E. O. (1984). Biophilia: Harvard University Press.

Younan, D., Tuvblad, C., Li, L., Wu, J., Lurmann, F., Franklin, M., . . Chen, J.-C. (2016). Environmental Determinants of Aggression in Adolescents: Role of Urban Neighborhood Greenspace. 
Journal of the American Academy of Child \& Adolescent Psychiatry, 55(7), 591-601. doi:http://dx.doi.org/10.1016/j.jaac.2016.05.002

Zelenski, J. M., \& Nisbet, E. K. (2014). Happiness and Feeling Connected The Distinct Role of Nature Relatedness. Environment and Behavior, 46(1), 3-23.

Zhang, J. W., Howell, R. T., \& Iyer, R. (2014). Engagement with natural beauty moderates the positive relation between connectedness with nature and psychological well-being. Journal of Environmental Psychology, 38, 55-63.

Zhang, J. W., Piff, P. K., lyer, R., Koleva, S., \& Keltner, D. (2014). An occasion for unselfing: beautiful nature leads to prosociality. Journal of Environmental Psychology, 37, 61-72. 